\title{
Absorption Spectra in the Detection of Chemical Changes in Cellulose and Cellulose Derivatives
}

\author{
By John W. Rowen, Charles M. Hunt, and Earle K. Plyler
}

\begin{abstract}
The spectral absorption of thin films of cellulose acetate, regenerated cellulose, the acetate and regenerated cellulose after oxidation with nitrogen dioxide gas, and the regenerated cellulose after oxidation with periodic acid followed by chlorous acid, are recorded for the infrared from 2 to 15 microns and the ultraviolet from 215 to 400 millimicrons. The regenerated cellulose absorbed ultraviolet radiant energy only at the short wavelength end of the region studied, and there the transmittance of a film 2.8 micons thick was only slightly reduced. The acetate and oxidized celluloses absorbed in this region to a greater extent but gave no narrow absorption bands. Marked changes were observed in some of the absorption bands in the infrared region in going from cellulose acetate to regenerated cellulose and to their oxidation products. These changes were correlated with changes in the $\mathrm{OH}, \mathrm{CO}$, and $\mathrm{COOH}$ groups. The results indicate the possibility of estimating the amount of these groups present by spectral absorption measurements
\end{abstract}

\section{Introduction}

Present-day theories regarding the chemistry and structure of cellulose, its derivatives and degradation products have been extensively studied and reviewed by several writers $[1,2,3,4,5,6] .^{1}$ However, only limited use of spectrometric measurements as a means of following the modification of cellulose by various treatments has been reported $[7,8,9,10]$. This paper contains results of the measurement of the absorption spectra of cellulose and cellulose acetate before and after chemical treatments.

The structural formula generally assigned to cellulose is:

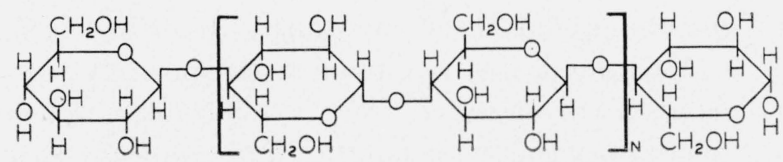

where $N$ may take values as high as 4,500. Any sample of cellulose is considered to be made up of varying proportions of these long-chain molecules, the lengths of the individual chains depending upon the source of cellulose and its history. The greater the proportion of the short chains in a sample, the greater the number of end groups.

\footnotetext{
* Published also in Textile Research Journal, Sept. 1947.

1 Figures in brackets indicate the literature references at the end of this paper.
}

Absorption Spectra in Cellulose
Cellulose is relatively unstable, and some of the linkages between atoms are ruptured, for example when cellulose is exposed to light, heat, or chemical agents. Shorter chains and additional end groups may be formed. When exposed to extreme conditions, cellulose may be degraded to relatively simple compounds.

The groups that are found in cellulose or which may be formed as a result of oxidation or cleavage include the ketone, aldehyde, hemiacetal, carboxyl, and hydroxyl groups. These groups are responsible for characteristic absorption bands in the spectra of simpler organic compounds. Not only would the bands be expected to be present in the absorption spectra of cellulose and cellulose derivatives, but the magnitude of the absorption might be a quantitative measure of the number of the groups responsible for the band.

Chemical methods for the quantitative evaluation of the groups in cellulosic materials $[5,15]$ sometimes lead to erroneous results because of changes in the cellulose produced by the conditions to which it is subjected during analysis. The spectrophotometric method of analysis probably would not result in appreciable change. Another way in which the spectrophotometric method could be useful is in investigating the preferential attack of reagents on specific parts of the cellulose 
molecule. For example, the oxidation of the secondary alcohol groups on carbon atoms 2 and 3 of the ring by periodic acid $[11,12]$ and the oxidation of the carbon atom in the 6 position to carboxyl by nitrogen dioxide gas [13, 14] could perhaps be followed spectrophotometrically.

The work reported in this paper was undertaken with this in mind.

\section{Materials and Methods}

The cellulose used in this study was prepared by deacetylation, in an inert nitrogen atmosphere, of cellulose acetate. The cellulose acetate was obtained in the form of yarns; it was first extracted with ether, dried, then wet with distilled water and dried again to insure complete removal of the ether. The acetate was dissolved in redistilled acetone and cast into films on a glass plate with a Bird film applicator, at room temperature. The film was then soaked from the plate with distilled water and dried.

\section{Preparation of Cellulose Films}

The films were deacetylated by soaking them for 24 hours in a $0.4 M$ alcoholic sodium hydroxide solution under an atmosphere of nitrogen. They were then washed with alcohol, soaked for 20 minutes in a 10-percent solution of glacial acetic acid in alcohol, again soaked for a half hour in water and then air-dried.

\section{Treatment of Films with Nitrogen Dioxide $\left(\mathrm{NO}_{2}-\mathrm{N}_{2} \mathrm{O}_{4}\right)$}

Films of cellulose acetate and/or regenerated cellulose were suspended at room temperature, in a glass chamber that was evacuated. Dry nitrogen dioxide was then passed into the chamber. The films were left in the nitrogen dioxide atmosphere for 2 hours and then soaked for another 2 hours in distilled water with three changes of water.

\section{Treatment of Films with Periodic Acid and Chlorous Acid}

The films were immersed for 2 hours in a 0.042 $M$ solution of periodic acid that was buffered with sodium acetate at a $\mathrm{pH}$ of 4.5. After contact with the periodic acid, the films were soaked for an additional 2 hours in distilled water and then washed three times with fresh distilled water. The films were then dried and subsequently transferred to a 5-percent solution of sodium chlorite buffered to a $\mathrm{pH}$ of 2.2 with phosphoric acid. They were allowed to remain in this solution for 2 hours. The films were then washed for 2 more hours and dried.

\section{Measurement of Thickness of Films}

Thickness measurements were made with a "Mikrokator" [16] that was checked against steel gage blocks calibrated by the Gage Section of the National Bureau of Standards. The standard deviation of five measurements did not exceed 0.2 $\mu$ on any spectrometer specimen. Thickness obtained in this manner with cellulose acetate film averaged $0.3 \mu$ less than thickness based on weight, area, density calculations.

\section{Measurement of Spectral Absorption in the Ultraviolet Region}

The ultraviolet absorption spectrum was measured with the Beckman model DU quartz spectrophotometer [17]. The wavelength calibrations on the scale of the instrument were found to check very well against the known wavelengths of the lines of the hydrogen- and mercury-discharge tubes. It was observed in the region from about 310 to $400 \mathrm{~m} \mu$ that when readings were made every $5 \mathrm{~m} \mu$, ripples appeared in the absorption pattern of these cellulosic materials. The amplitude and position of these ripples varied with the orientation of the sample in the light beam. They were interpreted as being caused by optical interference. It was possible to orient the film in the beam in such a position as to virtually eliminate them.

Figure 1 is based upon measurements made at $5 \mathrm{~m} \mu$ intervals.

\section{Measurement of Spectral Absorption in the Infrared Region}

The infrared absorption spectrum was measured with a Perkin-Elmer recording infrared spectrometer with a sodium chloride prism. This type of instrument has been previously described [18]. The instrument was equipped with specially constructed gears operated by a synchronous motor for changing the wavelength, and another gear mechanism for automatically increasing the slitwidth in such a way as to partially compensate for the lower energy of the radiation source in the longer wavelengths. As the instrument is a single-beam spectrometer, the absorption bands of atmospheric water vapor are superimposed upon the absorption spectrum of the film under observation. The estimation of the extent of absorption by the film in this region is therefore 


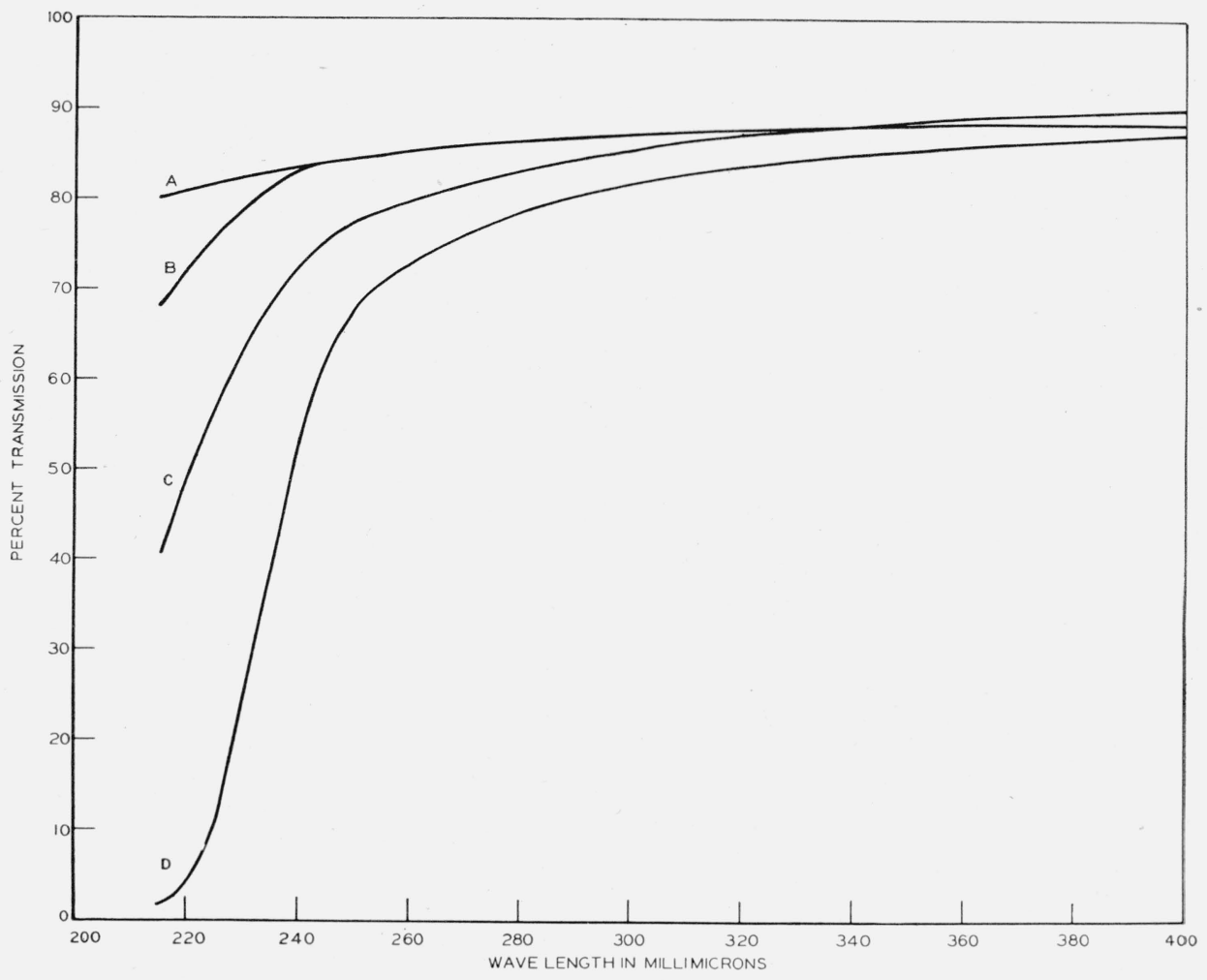

FiguRE 1.-Ultraviolet absorption curves.

$A$, Cellulose regenerated from cellulose acetate (film thickness $2.8 \mu$ ); $B$, cellulose treated with periodic acid followed by chlorous acid (film thickness $3.6 \mu$ ); $C$, ultraviolet absorption curve of cellulose acetate (film thickness $4.1 \mu$ ); $D$, cellulose treated with nitrogen dixoide gas (film thickness $3.3 \mu$ ).

less precise than in other regions. This uncertainty has been indicated in the spectra by broken lines.

\section{Results and Discussion}

The infrared and ultraviolet absorption spectra of cellulose, in the oxidized and acetylated states are shown in figures 1 to 6 inclusive. The infrared spectra of these materials have in general many more bands than the ultraviolet absorption spectra. The absorption of ultraviolet light by the resonance forms of the groups of the cellulose molecule appears to take place in the energy region that is mainly below $200 \mathrm{~m} \mu$ and hence in the vacuum region (see fig. 1). In order to properly evaluate these bands in the ultraviolet region it appears necessary to measure the absorption in the vacuum ultraviolet region as has been done for some aliphatic fatty acids [19]. It was noted that untreated cellulose had much less of a tendency to absorb short wavelength radiation than the modified celluloses as indicated in curve $A$ of figure 1. Exposure of such films of cellulose to most oxidizing agents, and light resulted in an increased absorption of the short wavelengths. In many cases the absorbing agent in the cellulose created by the treatment was water soluble and showed general absorption in water. In this way it was occasionally possible to check on the extent of oxidation and contamination of the films used in this study.

The groups in cellulose containing oxidized carbon gave rise to bands in the infrared region. This spectrum was, therefore, more informative than the ultraviolet absorption spectrum. The striking alteration that accompanies deacetylation may be noted by contrasting the curves of figure 2 and figure 4. The changes in the infrared absorption spectrum of cellulose acetate that occur upon exposure to nitrogen dioxide $\left(\mathrm{NO}_{2}-\mathrm{N}_{2} \mathrm{O}_{4}\right)$ may be seen in figures 2 and 3 . This oxidizing gas also has a marked effect on the absorption spectrum of cellulose. The effect of a somewhat arbitrary concentration of periodic acid (followed by chlorous acid) on the infrared absorption spectrum of cellulose is shown in figure 6 . The bands shown in the various figures are discussed under the appropriate title as follows: 

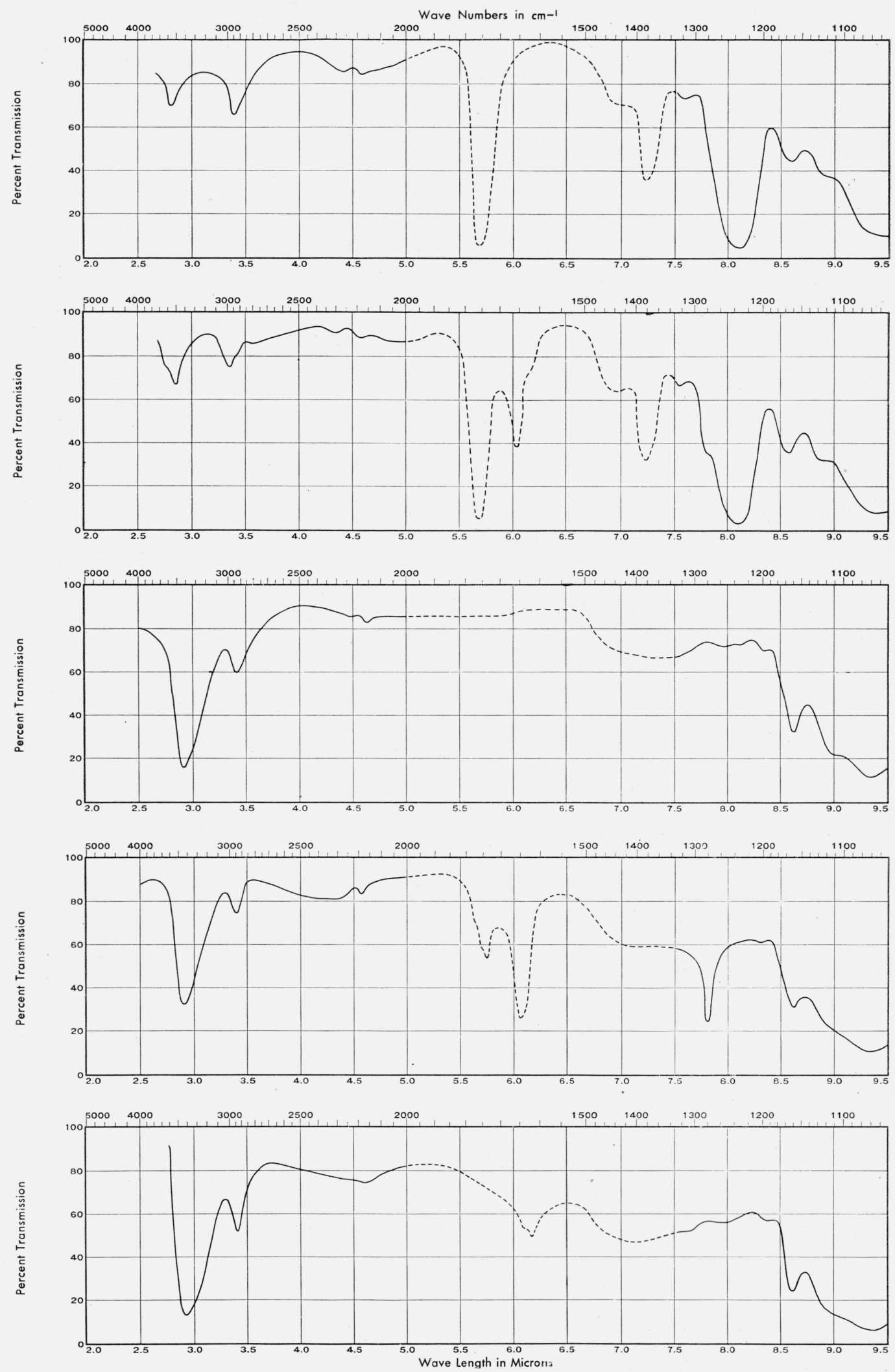


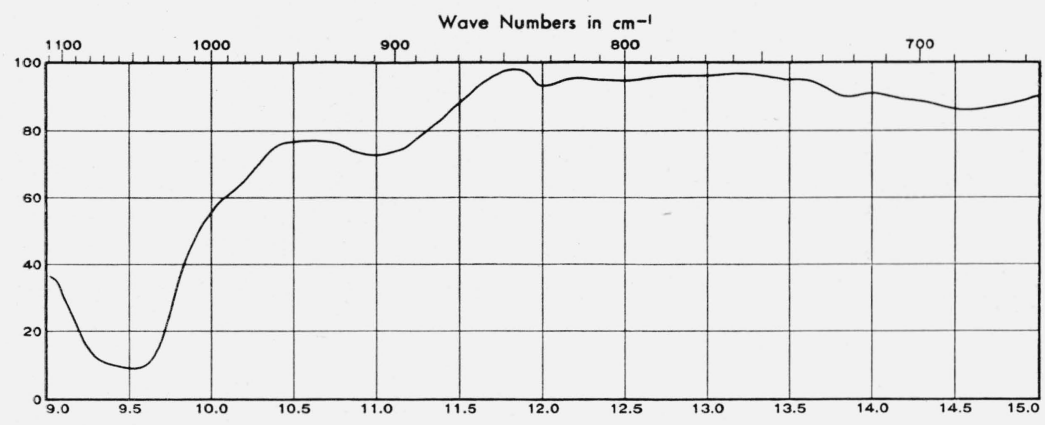

Figure 2.-Infrared absorption curve of cellulose acetate (film thickness $4.1 \mu$ ).

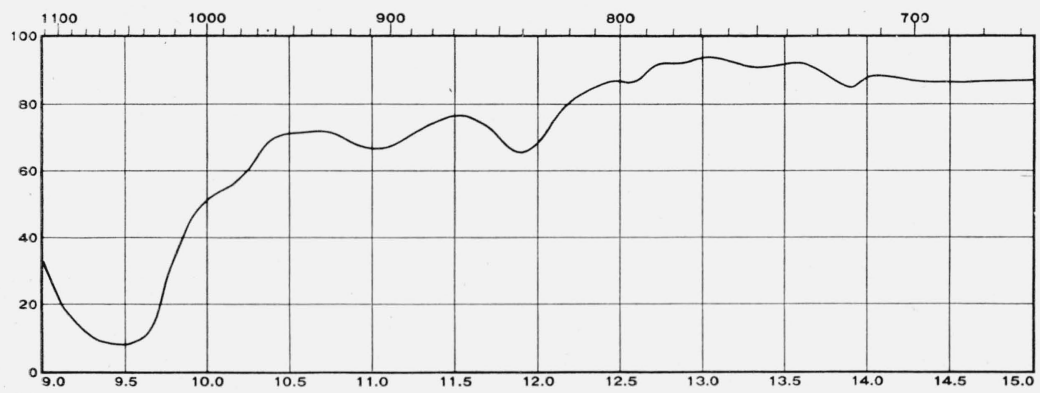

Figure 3.-Infrared absorption curve of cellulose acetate exposed to nitrogen dioxide gas (film thickness $3.3 \mu$ ).

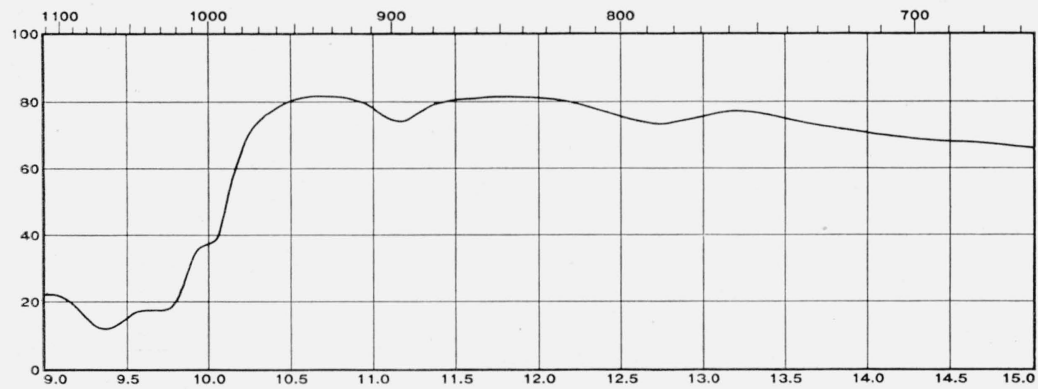

Figure 4.-Infrared absorption curve of regenerated cellulose (film thickness $2.8 \mu$ ).

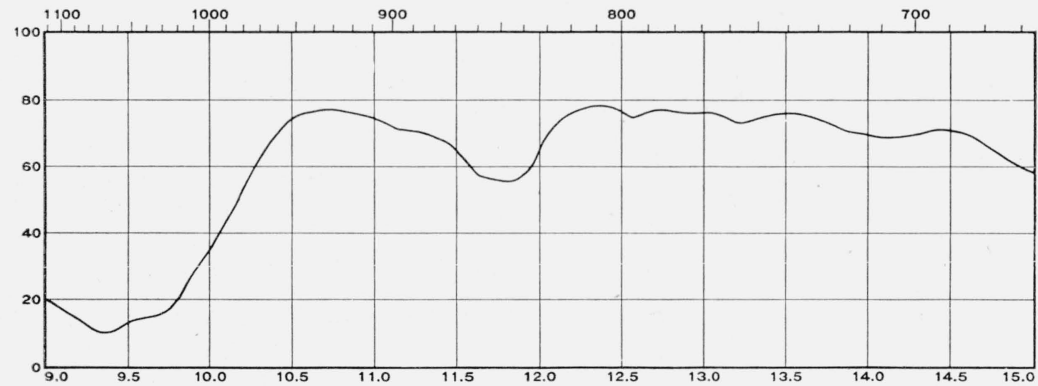

Figure 5.-Infrared absorption curve of regenerated cellulose exposed to nitrogen dioxide gas (film thicknes: $3.3 \mu)$.

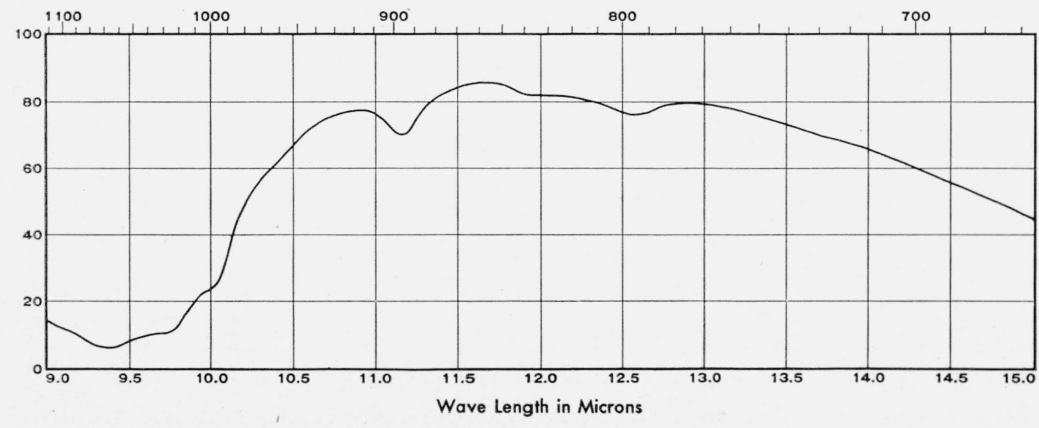

Figure 6.-Infrared absorption curve of regenerated cellulose treated with periodic acid followed by chlorous acid (film thickness $3.6 \mu$ ). 


\section{Cellulose Acetate}

As indicated under the experimental part, the cellulose acetate was the starting point for the regeneration of the cellulose used in the study. The infrared absorption spectrum of the original cellulose acetate is shown in figure 2. This spectrum is characterized by several well-defined bands. The first band of appreciable intensity occurs at $2.8 \mu$ and is probably related to the $\mathrm{O}-\mathrm{H}$ stretching vibration. The band at $3.4 \mu$ is believed to be the familiar band attributed to the $\mathrm{C}-\mathrm{H}$ stretching vibration. The intense band at $5.7 \mu$ falls in the general region characteristic of the ester carbonyl group, and it shows a strong absorption in the cellulose acetate film. The band at $7.2 \mu$ is characteristic of the $\mathrm{C}-\mathrm{CH}_{3}$ group and hence appears in both figures 2 and 3 (untreated and nitrogen dioxide treated cellulose acetate). The intense absorption at $8.1 \mu$ is also present in curves 2 and 3 . It is attributed to the ester group, as a band in this general position is always observed in the spectra of esters. These last three bands also appear in polyvinyl acetate as well as in other simpler acetates [20]. The strong band at $9.5 \mu$ is present in all the infrared absorption spectra (figures 2 to 6 inclusive) and is probably due to the $\mathrm{C}-\mathrm{O}$ vibration. This vibration is very intense for methyl alcohol and is also found in polyvinyl alcohol [20].

\section{Oxidized Cellulose Acetate}

When cellulose acetate is oxidized with nitrogen dioxide $\left(\mathrm{N}_{2} \mathrm{O}_{4}-\mathrm{NO}_{2}\right)$ two new bands (fig. 3) appear in the infrared spectrum. These absorptions take place at 6.1 and $7.8 \mu$. The band at $7.8 \mu$ is evident as a bulge in the side of the broad band at 8.1 $\mu$. It will be noted later that these two bands correspond closely with the two bands that are created when cellulose is treated with nitrogen dioxide. Most organic compounds containing carboxyl groups have two strong absorption bands at approximately 6.0 and $7.8 \mu$. The possibility that the two bands described above might be due to carboxyl groups created by the oxidation of the unacetylated number six $\mathrm{C}-\mathrm{OH}$ group will be discussed later.

\section{Cellulose}

When cellulose acetate is deacetylated, the marked changes that result from this process may be noted by comparing figure 2 with figure 4 . One notes that the $\mathrm{O}-\mathrm{H}$ vibration at $2.8 \mu$ has become much more intense and at the same time slightly shifted to longer wavelengths. The ester carbonyl group at $5.7 \mu$, the $\mathrm{CH}_{3}$ group at 7.2 and the typical ester band at $8.1 \mu$ are all absent. On the other hand the $\mathrm{C}-\mathrm{O}$ band at $9.5 \mu$ has been considerably broadened.

\section{Cellulose Oxidized With Nitrogen Dioxide}

When the cellulose film is treated (as described under experimental procedure) with nitrogen dioxide, certain characteristic changes occur in the spectrum of cellulose, figure 5 . First the $\mathrm{OH}$ absorption at $2.9 \mu$, seen in figure 4 , is somewhat decreased in intensity. In addition, three other bands appear, the one at 5.7 , one at 6.1 , and a third at $7.8 \mu$. The band at $5.7 \mu$ falls in the general region characteristic of the $\mathrm{C}=\mathrm{O}$ group, and the other two bands are also associated with the carboxyl group as indicated under the discussion of oxidized cellulose acetate. In this work, the two strong bands at 6.1 and $7.8 \mu$ were always associated with the oxidized cellulose believed to contain carboxyl groups.

\section{Cellulose Treated With Periodic and Chlorous Acids}

Exploratory experiments with the effect of periodic and chlorous acids on the infrared absorption spectrum of cellulose have been performed. Neither of these acids alone or in sequence had nearly so marked an effect on the cellulose as had nitrogen dioxide. However, a small band shown in figure 6 at $6.2 \mu$, not present in the spectrum of the original cellulose, was found in the treated cellulose. No definite conclusions can be drawn concerning the band until more information is available.

\section{Relationship Between Spectra and Structures}

Cellulose contains over 49 percent of oxygen, and this oxygen content may be expected to undergo a very small but definite increase throughout the life of the cellulose in the atmosphere. The oxidized cellulose would not be expected to be a homogeneous molecular species. In fact, one would expect the oxidized cellulose to consist of a mixture of many different kinds of oxidized cellu- 
lose molecules. Workers recognizing the complexity of "oxidized" cellulose have attempted to study the effect of specific oxidizing agents on the polymer. Kenyon and coworkers have studied the oxidation of cellulose by nitrogen dioxide. They presented evidence $[13,14]$ that the mode of attack by this agent was highly specific. Their work indicated that the primary alcohol group on the number six carbon atom was oxidized to a carboxyl group by the oxidizing gas. The work of Jackson and Hudson [11], and Harris et al [12] indicated that periodic acid has a specific oxidizing effect on cellulose. These experiments indicate that this agent converts the secondary alcohol groups on carbons 2 and 3 to aldehyde groups in accordance with the following scheme:

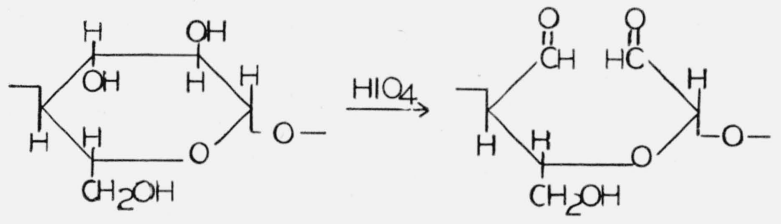

As the conditions of the reaction of nitrogen dioxide with cellulose, described under the experimental part are very similar to those employed by Kenyon et al $[13,14]$, it is reasonable to suppose that carboxyl groups were the primary product of the reaction. Furthermore, as adequate steps had been taken to remove any unreacted nitrogen dioxide, and as very little if any nitrocellulose would be formed under the experimental conditions, it appears reasonable to assume that the three bands present in figure 5 (the ones at 5.7, 6.1 and $7.8 \mu$ ) are all manifestations of the carboxyl group in the number six position.

Whether or not the intensities of these bands lend themselves to the quantitative estimation of carboxyl groups is not known at present. The presence of the "carboxyl" bands (5.7, 6.1, and $7.8 \mu)$ in the spectrum of the treated cellulose acetate films (fig. 3) suggests the possibility that a good number of the primary alcohol groups (number six position) present in this material are attacked by nitrogen dioxide and converted to carboxyl groups.

The intensification of the $\mathrm{O}-\mathrm{H}$ band $(2.8 \mu)$ in figure 4 that accompanies deacetylation is quite striking. Presumably each acetyl group (absorption at $5.7,7.2$, and $8.1 \mu$ ) is replaced by a hydroxyl group in this conversion, and the difference in intensities in the two bands ( 2.8 to 2.9 microns) is some measure of the number of $\mathrm{OH}$ groups regenerated in the process.

The effect of periodic acid, reported by other workers, was not observed in the absorption spectra of the treated cellulose in the spectral ranges studied. However, it is pointed out that these experiments may not have provided the optimum conditions for the generation of the aldehyde groups on the second and third carbon atoms. The weak band at $6.2 \mu$ in figure 6 is evidence of some reaction that did take place in these preliminary attempts.

When the cellulose acetate is converted to cellulose, and when the latter is oxidized to oxycellulose, certain fundamental aspects of the long chain molecular structure remain intact. For example, the skeletal ring-chain structure should not be profoundly altered during some of the above reaction steps. One might, therefore, expect to see manifestations of the unchanging portions of the molecule in the infrared spectrum. The absorption band at $8.6 \mu$ appears in the spectrum of all of the cellulose derivatives examined in this study. This band may be a fundamental vibration that is associated with the ring skeleton. The absorption band at $3.4 \mu$ also is common to all of the spectra, and it is undoubtedly due to the $\mathrm{C}-\mathrm{H}$ stretching vibration that is characteristic of all of the compounds.

This study indicates the possibility of using the infrared absorption spectrum as a means of elucidating the alterations that may be made in the cellulose molecule.

\section{Summary}

1. The ultraviolet and infrared absorption spectra of cellulose, cellulose acetate, and certain oxidized samples of cellulose have been obtained.

2. Acetylation and oxidation of cellulose are reflected by profound changes in the absorption spectra of these materials.

3. This study indicates the possibility of detecting and estimating the amounts of such groups as

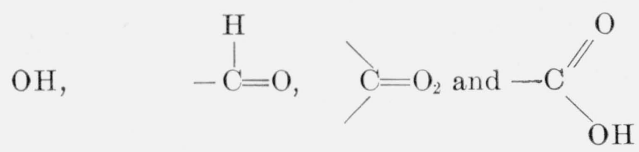

in cellulosic materials. 
The authors are indented to Lee A. Dunlap and Walter Stone for assistance in making some of the measurements reported in this paper.

\section{References}

[1] Emil Ott, Cellulose and cellulose derivatives (Interscience Publishers, Inc., New York, N. Y., 1943)

[2] Emil Heuser, The chemistry of cellulose (John Wiley \& Sons, Inc. New York, N. Y., 1944).

[3] G. F Davidson, J. Textile Inst. 27, 144 (1936).

[4] W. N. Haworth J. Dyersand Colourists 56 49 (1940).

[5] C. C. Unruh and W. O. Kenyon, Textile Research J. 16, 1 (1946).

[6] E. Pacsu and L. A. Hiller, Textile Research J. 16, 243 (1946).

[7] R. O. Herzog and G. Laski, Z Physik Chem. 121, 136 (1926).

[8] A. J. Wells, J. Appl. Phys. 11, 137 (1940).

[9] G. Champetier and R. Marton, Bul. Soc. Chem,, France 10, 102 (1943).

[10] Katsumoto Arsuki, and Hiroshi, Sobue, J. Soc. Chem. Ind., Japan Suppl. Binding 36, 589B (1933)
[11] E. L. Jackson and C. S. Hudson, J. Am. Chem. Soc. 59, 2049 (1937); 60, 989 (1938).

[12] H. A. Rutherford, F. W. Minor, A. R. Martin, and M. Harris, J. Research NBS 28, 131 (1942) RP1491.

[13] E. C. Yackel and W. O. Kenyon, J. Am Chem. Soc. 64, 121 (1942).

[14] C. C. Un:'uh and W. O. Kenyon, I. Am. Chem. Soc 64. 127 (1942).

[15] A. M. Sookne and M. Harris, Cellulose and cellulose derivatives p. 77 (Interscience Publishers, Ine., New York, N. Y., 1943).

[16] Machinery (New York) 45, 729 (June 1939) : 46, 127 Feb. 1940)

[17] H. H. Cary and A. O Beckman, J Optical Soc. Am. 31, 682 (1940).

[18] R. Bowling Barnes, R. S. MeDonald, van Zandt Williams, and R. F. Kinnaird, J. Appl. Phys. 16, 77 (1945).

[19] J. R. Platt I. Rusoff, and H. B. Klevens, J Chem. Phys. 11, 535 (1943).

[20] R. B. Barnes, R. C. Gore, U. Liddel, and van Z. Williams, Infrared spectroscopy (Reinhold Publishing Co., New York, N. Y., 1944).

Washington, May 6,1947 